\title{
GC/MS Analysis of Fatty Acids on Pliek $U$ Oil and Its Pharmacological Study by Molecular Docking to Filaggrin as a Drug Candidate in Atopic Dermatitis Treatment
}

\author{
Nanda Earlia, ${ }^{1,2}$ Muslem, ${ }^{3}$ Rivansyah Suhendra ${ }^{\mathbb{D}},{ }^{4}$ Mohamad Amin, ${ }^{5}$ C. R. S. Prakoeswa, \\ Khairan, ${ }^{7}$ and Rinaldi Idroes $\mathbb{D}^{3,7}$ \\ ${ }^{1}$ Graduate School of Mathematics and Applied Sciences, Universitas Syiah Kuala, Banda Aceh 23111, Indonesia \\ ${ }^{2}$ School of Medicine, Universitas Syiah Kuala, Banda Aceh 23111, Indonesia \\ ${ }^{3}$ Department of Chemistry, Faculty of Mathematics and Natural Sciences, Universitas Syiah Kuala, Banda Aceh 23111, Indonesia \\ ${ }^{4}$ Department of Informatics, Faculty of Mathematics and Natural Sciences, Universitas Syiah Kuala, \\ Banda Aceh 23111, Indonesia \\ ${ }^{5}$ Department of Biology, Faculty of Mathematics and Natural Sciences, Universitas Negeri Malang, Malang 65145, Indonesia \\ ${ }^{6}$ Faculty of Medicine, Universitas Airlangga-Dr Soetomo, General Academic Hospital, Surabaya 60132, Indonesia \\ ${ }^{7}$ Department of Pharmacy, Faculty of Mathematics and Natural Sciences, Universitas Syiah Kuala, Banda Aceh 23111, Indonesia
}

Correspondence should be addressed to Rinaldi Idroes; rinaldi.idroes@unsyiah.ac.id

Received 2 July 2019; Accepted 5 September 2019; Published 3 November 2019

Academic Editor: Rizwan Hasan Khan

Copyright $\odot 2019$ Nanda Earlia et al. This is an open access article distributed under the Creative Commons Attribution License, which permits unrestricted use, distribution, and reproduction in any medium, provided the original work is properly cited.

Analysis of fatty acid contents and pharmacological properties of Pliek $U$ oil was performed. Fatty acids were analyzed by gas chromatography-mass spectrometry (GC-MS), and pharmacological properties based on its potential on filament-aggregating protein (filaggrin) were studied with bioinformatics approach by the reverse docking technique using palmitic acid as a control compound. Two Pliek $U$ extracts, namely, Pliek $U$ oil (PUO) and ethanolic Pliek $U$ oil extract (EPUOE), were prepared. The GCMS results revealed that lauric acid, myristic acid, palmitic acid, and oleic acid are the predominant fatty acids, with lauric acid being the abundant one in all Pliek $U$ oil extracts. The reverse docking technique results showed that oleic acid had the most stable interaction to filaggrin with the lowest binding affinity $(-6.1 \mathrm{kcal} / \mathrm{mol})$. Oleic acid and palmitic acid have one same side binding to filaggrin on amino acid LEU D75. These findings indicated that oleic acid has the best potential to be used as a drug candidate in atopic dermatitis treatment.

\section{Introduction}

Skin has two main functions; it acts as an external barrier and provides protection to the body. Skin hinders excessive water loss from the tissue and at the same time protects the body against foreign pathogens. The skin layer that acts as the protection barrier is the widely bound lipid-protein solid matrix called stratum corneum. The primary protein of the lipid-protein matrix is filaggrin [1], while the lipid consisted of ceramides, fatty acids, and cholesterol [2].

Structural damages to the lipid-protein matrix may result in major disfunction of the skin barrier-protection system. Studies on atopic dermatitis reveal damages to filaggrin $[1,3,4]$ and deficiency of free fatty acids in the lipid structure as the main causes of diseases [2]. Deficiency of fatty acids in the lipid structure leads to the reduction of skin humidity that causes dry skin. This further causes itching that triggers mechanical damage of the skin barrier-protection system $[5,6]$.

One of the treatments used to cure atopic dermatitis is applying the moisturizing cream. The moisturizer works to soothe dryness and itching as well as to recover the damaged barrier-protection system. Fatty acids are the main components of moisturizing cream due to their humectant and 
occlusive effects. Fatty acids also offer pharmacological benefits to repair skin barrier-protection and to accelerate wound healing through rapid epithelization [7-9].

Fatty acids can be found in animal fats and vegetable oils that can be extracted from fruits, seeds, and nuts [10]. Coconut meat has a considerable amount of fatty acids in it. In addition, coconut has been notably identified as a commodity of tropical countries. In Indonesia, especially in Aceh province, coconut is harvested by local farmers, and its meat is traditionally processed through fermentation to produce coconut oil (commonly known as Pliek $U$ oil) [7]. Pliek $U$ oil is known for its rich fatty acid content, thus allowing this fermented product to be not only treated as a food product but also as a medical potential. Further development of this product, especially as a medical potential, can economically help the local farmers.

However, further studies on the fatty acid content of coconut meat products (such as coconut oil) are required. This is due to the fact that the fatty acid content can be varied depending on the geographical condition of the cultivation, the varieties [11], and the harvesting time [12]. Therefore, it is important to conduct studies that determine the predominant fatty acid that can provide a reference for the development of medical potential of coconut meat products.

Studies on medicinal compound activities against certain proteins generally are done by spectroscopy approaches such as UV-Vis absorption [13], sensor [14], laser, and electrometric methods $[15,16]$, but today it is carried out predominantly by computational methods, either virtually or in silico $[17,18]$. Virtual screening with molecular docking is one of the computational methods used for the said purpose. Molecular docking studies are carried out on the structural basis of the screened-potential medicinal compound against the complementary macromolecular binding sides with its protein (structure orientation and electrostatic interaction) [19]. A fatty acid that has the most suitable and stable structural orientation to electrostatically interact with filaggrin protein is then suggested as the highly effective medicinal compound.

In this research, the fatty acids of Pliek $U$ oil were analyzed with gas chromatography-mass spectrometry (GCMS) and further analyzed with the reverse docking technique to evaluate its potential medicinal activities.

\section{Materials and Methods}

2.1. Sample Extraction. Coconut fruit samples were obtained from the Pliek $U$ raw material supplier in Matang subdistrict, Bireuen district, Aceh province. The samples were prepared to produce Pliek $U$ oil (PUO) and ethanolic Pliek $U$ oil extract (EPUOE).

The coconut fruits were cleaved and the water was drained off. The fruits were left to dry for 5 days. The meat was grated and placed in a sealed container. The meat was left for another 5 days at room temperature $\left(29-36^{\circ} \mathrm{C}\right)$ and then fermented. The fermented coconut meat was then squeezed to release the oil. Next, the oil is collected and filtered. At the end of this procedure, the Pliek $U$ oil is produced and labeled as PUO.
A separating funnel was filled with $250 \mathrm{ml}$ of Pliek $U$ oil and $300 \mathrm{ml}$ of $96 \%$ ethanol. The ethanol fraction was separated into a Baker glass; $300 \mathrm{ml}$ of $96 \%$ ethanol was added back into the separating funnel. The ethanol fraction was separated and collected with the previous fraction. The ethanol fraction was then concentrated with a rotary evaporator at $50^{\circ} \mathrm{C}$. This procedure gives ethanolic Pliek $U$ extract, which is labeled as EPUOE.

2.2. Sample Preparation for GC-MS. For GC-MS analysis, 1 gram of PUO and EPUOE extracts were firstly dissolved into $n$-hexane p.a. and vortexed for 2 minutes, respectively. Then, the hexane phase was separated and moved into the derivation tube and then dried with nitrogen bursts. After that $2 \mathrm{ml}$ of $2 \% \mathrm{NaOH}(\mathrm{NaOH}$ in methanol) was added, sealed tightly, and heated at $90^{\circ} \mathrm{C}$ for 5 minutes, and it was left until cold. $2 \mathrm{ml}$ of $\mathrm{BF}_{3}$ in methanol was added, sealed tightly, and reheated for 30 minutes. After being left to cool down, it was extracted with $3 \mathrm{ml} n$-hexane p.a. The $n$-hexane phase (upper phase) was taken for GC-MS analysis.

2.3. GC-MS Data Analysis. The peaks of the chromatogram were identified based on MS data analysis to determine the fatty acid content. The percentage of each fatty acid was determined by area percentage (\%) of each peak, which later was employed in the determination of predominant fatty acid of each sample.

2.4. Docking Procedure. The 3D structure of control and target compounds was downloaded from PubChem database. Meanwhile, the 3D structure of the protein was downloaded from the database of Protein Data Bank (GDP). Then, the natural ligand on the target protein was cut using PyMOL software (https://pymol.en.softonic.com/?ex=DSK1262.10). After that, the docking process between the control compound and prediction compound of the target protein was carried out using PyRx computer software (https://pyrx. sourceforge.io/downloads).

2.5. Visualization of Docking Results. Visualization of docking result was performed by PyMOL and Discovery Studio software (https://www.3dsbiovia.com/products/ collaborative-science/biovia-discovery-studio/visualizationdownload.php).

\section{Results and Discussion}

3.1. Fatty Acid Analysis of Pliek U Oil by GC-MS. The analyzed data of GC-MS, either for PUO or EPUOE, indicate similar predominant fatty acid content. It is observable that each sample has a different percentage of fatty acids. It can be seen from Table 1 that the highest fatty acid content is in lauric acid $(\mathrm{PUO}=30.13 \%$ and $\mathrm{EPUOE}=36.95 \%)$, followed by myristic acid (PUO $=22.25 \%$ and $\mathrm{EPUOE}=23.55 \%$ ), palmitic acid (PUO $=15.460 \%$ and $\mathrm{EPUOE}=12.925 \%$ ), oleic acid $(\mathrm{PUO}=13.476 \%$ and $\mathrm{EPUOE}=9.980 \%)$, stearic acid $(\mathrm{PUO}=9.337 \%$ and $\mathrm{EPUOE}=5.722 \%)$, capric acid $(3.063 \%$ 
and 4.433), linoleic acid (3.367\% and 2.257\%), caprylic acid (2.281\% and 3.397\%), and elaidic acid (0.363\% and $0.323 \%)$.

Based on the GC-MS analysis, the predominant fatty acid in Pliek $U$ oil in this research was similar to the predominant fatty acids found in the coconut oil reported [20]. The predominant composition of fatty acids in coconut oil per the GC-MS results was lauric acid (47.7\%), myristic acid (19.9\%), caprylic acid (7.6\%), and oleic acid (6.2\%). The difference in predominant fatty acid contents was only in palmitic acid, where it was found to be higher in Pliek $U$ oil. This difference was probably associated with the geographical factor, where the coconuts were cultivated (Laureles, 2002). Based on these data of fatty acid contents, Pliek $U$ oil possibly has a significant potential to be utilized for medical purposes, particularly for atopic dermatitis treatment.

\subsection{Molecular Docking Results Based on Affinity Binding} Value. The common virtual docking of PyRx software was used to dock the 3D structure of ligands (caprylic, capric, lauric, linoleic, myristic, and oleic acids) and the control ligand (palmitic acid) to the target protein (filaggrin). The result of this molecular docking process is the affinity binding value between each ligand to the target protein presented in Table 2.

The binding affinity between fatty acids and filaggrin showed that only oleic acid had a lower binding affinity $(-6.1 \mathrm{kcal} / \mathrm{mol})$ compared with the control ligand of palmitic acid $(-5.9 \mathrm{kcal} / \mathrm{mol})$. The other ligands showed a greater binding affinity than palmitic acid. The low binding affinity value is associated with the interaction stability between the ligand and the protein. The low binding affinity indicates that the interaction between the ligand and the protein requires low energy. Gibbs's energy theory states that the smaller the energy generated from a bond between the ligand and its receptor, the more stable the bond is [14]. Therefore, oleic acid had the most stable bond to bind filaggrin, in comparison with other fatty acids. In drug design, the stability of interaction between a drug (ligand) and a receptor (target protein) is important to identify the molecular and macromolecular interactions because they determine the therapeutic effects. This information is crucial for the dosage of medicine so that medical treatment is in accordance with what is expected.

\subsection{Visualization of Fatty Acid and Filaggrin Interaction.} Interaction visualization with molecule attachment was conducted to observe the interaction similarity of the control ligand (palmitic acid) and the prediction ligand (caprylic, capric, lauric, linoleic, myristic, and oleic acids) with the target protein (filaggrin). The visualization was carried out with PyMOL software. The obtained information includes the type and the position of the bonding/interaction between the ligand and filaggrin. The optimum interaction was determined based on the interaction similarity between the control ligand with filaggrin and prediction ligand with filaggrin, as well as the number of ligand interaction on protein binding sides. Palmitic acid interaction and filaggrin
TABLe 1: Fatty acid analysis of PUO and EPUOE by GC-MS.

\begin{tabular}{lcc}
\hline Component & PUO (\%) & EPUOE (\%) \\
\hline Caprylic acid & 2.281 & 3.397 \\
Capric acid & 3.063 & 4.433 \\
Lauric acid & 30.130 & 36.959 \\
Myristic acid & 22.255 & 23.557 \\
Palmitic acid & 15.460 & 12.925 \\
Linoleic acid & 3.367 & 2.257 \\
Oleic acid & 13.476 & 9.980 \\
Elaidic acid & 0.363 & 0.323 \\
Stearic acid & 9.337 & 5.722 \\
\hline
\end{tabular}

TABle 2: Fatty acid binding affinity of PUO and EPUOE samples.

\begin{tabular}{lcc}
\hline No & Ligand & Binding affinity $(\mathrm{kcal} / \mathrm{mol})$ \\
\hline 1 & Capric acid & -5.2 \\
2 & Caprylic acid & -4.8 \\
3 & Lauric acid & -5.2 \\
4 & Linoleic acid & -5.7 \\
5 & Myristic acid & -5.6 \\
6 & Oleic acid & -6.1 \\
7 & Palmitic acid & -5.9 \\
\hline
\end{tabular}

formed through hydrogen bond and hydrophobic interaction. The hydrogen bond was formed on the THR D71 amino acid residue, while hydrophobic interaction was formed on amino acid residues MET A76, ILE D62, LEU A80, LEU A44, PHE A56, and LEU D75.

3.3.1. Visualization of Capric Acid and Filaggrin Interaction. Capric acid interacts with filaggrin (Figure 1) on the GLU D72 amino acid residue through hydrogen bond and ILE D62, LYS A76, and LEU D75 through hydrophobic interactions. Therefore, capric acid and palmitic acid had two same side interactions to filaggrin, namely, ILE D62 and LEU D75.

\subsubsection{Visualization of Caprylic Acid and Filaggrin} Interaction. The visualization results between caprylic acid and filaggrin (Figure 2) showed the presence of hydrogen bonds and hydrophobic interactions. Hydrogen bonds occur on amino acids ASP D69 and ASP D63, and meanwhile the hydrophobic interaction occur on PHE A56, MET A76, LEU A44, LEU A80, and ILE D62 amino acid residues. Based on the amino acid residues of palmitic acid and caprylic acid, filaggrin is bound on the same sides on the amino acid residues PHE A56, MET A76, LEU A44, and ILE D62.

\subsubsection{Visualization of Lauric Acid and Filaggrin Interaction.} The interaction between lauric acid and filaggrin (Figure 3) only occurs through hydrophobic interactions. The interaction happened on amino acid residues HIS A59, PHE A56, and ILE D62. Therefore, capric acid and palmitic acid had two similar interaction sides to filaggrin, namely, PHE A56 and ILE D62. 


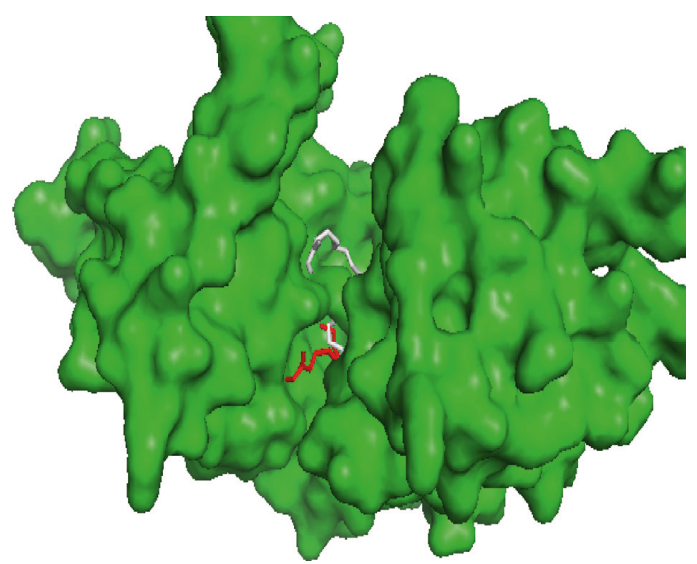

(a)

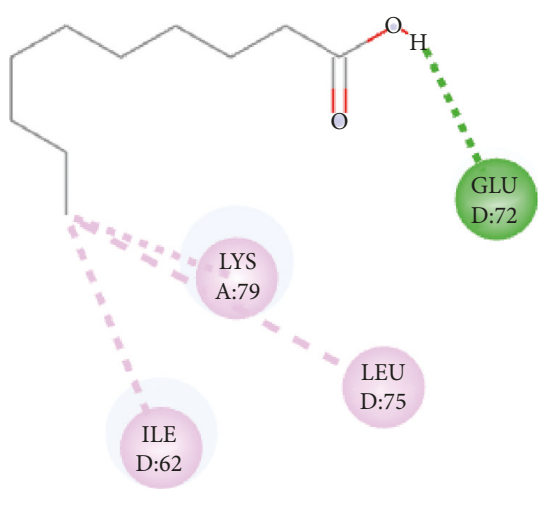

(b)

Figure 1: Interaction of capric and palmitic acids with filaggrin. (a) 3D visualization: capric acid (red) and palmitic acid (grey). (b) 2D visualization: amino acid residues in capric acid and palmitic acid.

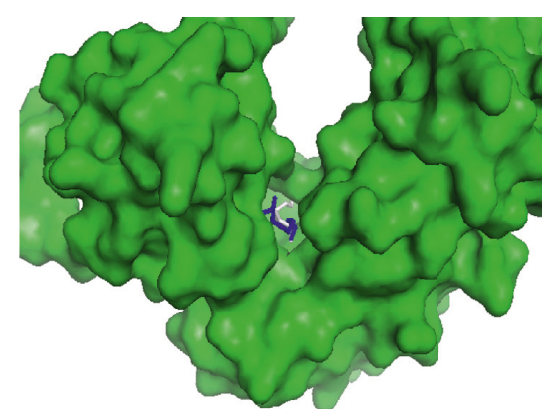

(a)

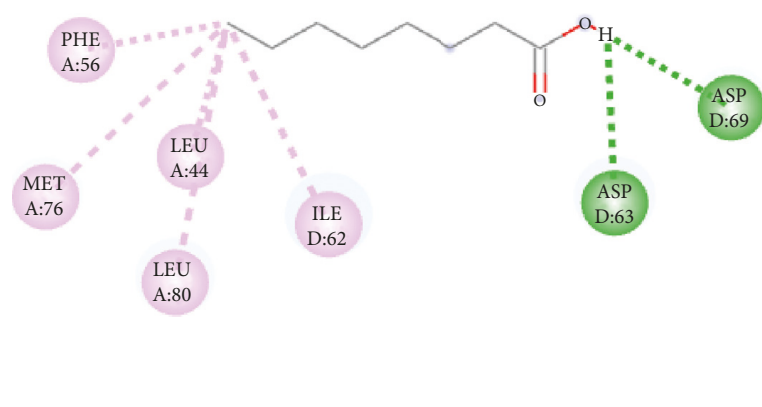

(b)

FIgURE 2: Interaction of caprylic and palmitic acids with filaggrin. (a) 3D visualization: caprylic acid (blue) and palmitic acid (grey). (b) $2 \mathrm{D}$ visualization: amino acid residues in caprylic acid and palmitic acid.

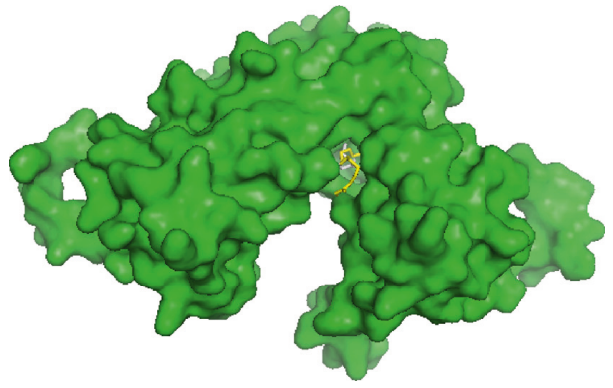

(a)

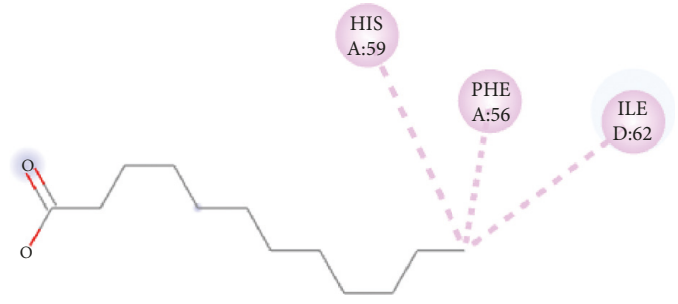

(b)

FIgURe 3: Interaction of lauric and palmitic acids with filaggrin. (a) 3D visualization: lauric acid (yellow) and palmitic acid (grey). (b) 2D visualization: amino acid residues in lauric acid and palmitic acid.

3.3.4. Visualization of Linoleic Acid and Filaggrin Interaction. Linoleic acid interacts with filaggrin (Figure 4) on amino acid residue LYS A76 through hydrogen bond and amino acid residue ALA A83 through hydrophobic interaction. Therefore, linoleic acid and palmitic acid do not have any same side interaction with filaggrin.

3.3.5. Visualization of Myristic Acid and Filaggrin Interaction. The visualization results between the myristic acid and filaggrin (Figure 5) showed the presence of a mere hydrophobic interaction. The interaction occurred on amino acid residues ILE D62 and LEU D75. Based on the fact, palmitic acid, myristic acid, and palmitic acid bound filaggrin on the same sides of amino acid residues, namely, ILE D62 and LEU D75.

3.3.6. Visualization of Oleic Acid and Filaggrin Interaction. The interaction between oleic acid and filaggrin (Figure 6) occurs only through hydrophobic interactions. The 


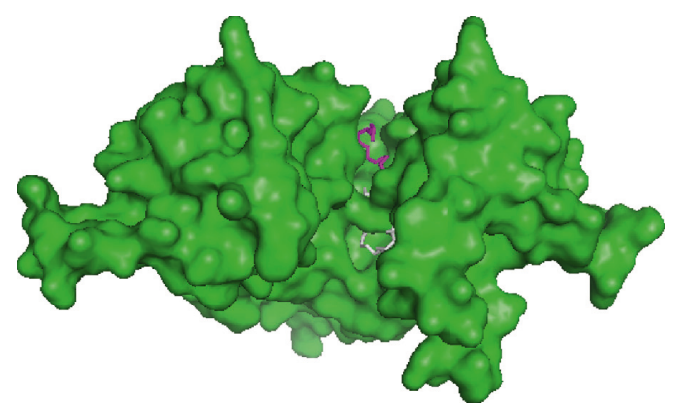

(a)

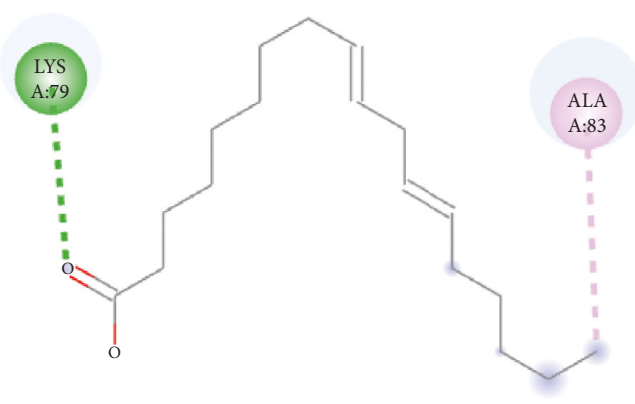

(b)

FIGURE 4: Interaction of linoleic and palmitic acids with filaggrin. (a) 3D visualization: linoleic acid (purple) and palmitic acid (grey). (b) 2D visualization: amino acid residues in linoleic acid and palmitic acid.

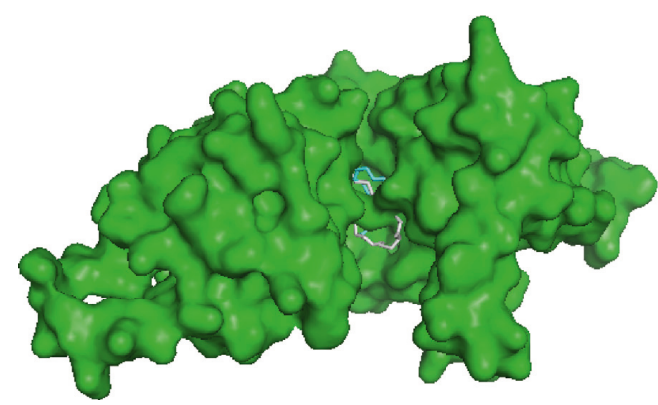

(a)

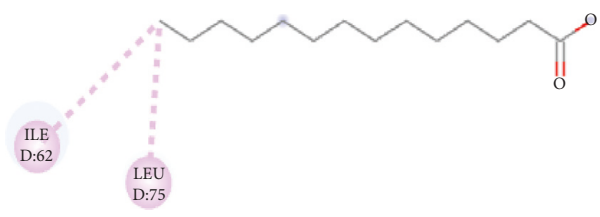

(b)

Figure 5: Interaction of myristic and palmitic acids with filaggrin. (a) 3D visualization: myristic acid (light blue) and palmitic acid (grey). (b) 2D visualization: amino acid residues in myristic acid and palmitic acid.

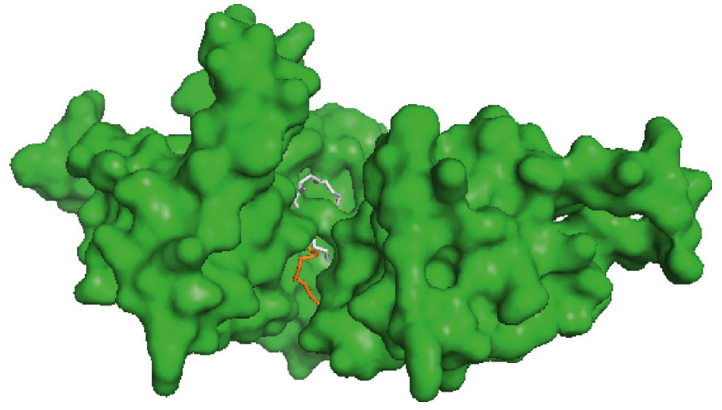

(a)

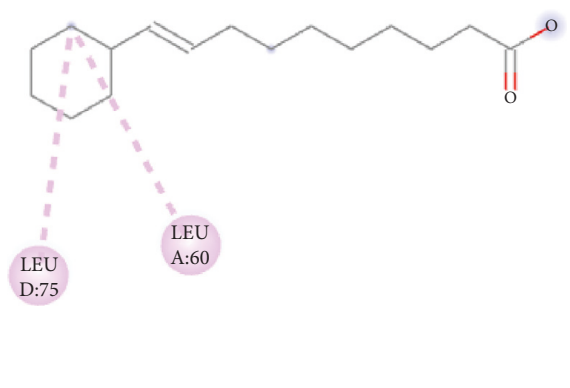

(b)

Figure 6: Interaction of oleic and palmitic acids with filaggrin. (a) 3D visualization: oleic acid (orange) and palmitic acid (grey). (b) 2D visualization: amino acid residues in oleic acid and palmitic acid.

interactions were found to be on amino acid residues LEU A60 and LEU D75. Therefore, oleic acid and palmitic acid have one similar interaction side to filaggrin, which is amino acid residue LEU D75.

Visualization of the interaction between fatty acids (predicted ligand) and filaggrin (protein target) reveals that all fatty acids and palmitic acid (control ligand) have the similar binding side to filaggrin, except linoleic acid. Especially for caprylic acid, it has four same binding sides with palmitic acid, which is quantitatively the highest one among the other fatty acids. Based on this result, it can be concluded that caprylic acid has the best structural conformation to bind filaggrin with the best orientation of interaction.

According to the molecular docking analysis, oleic acid gave the most stable interaction with filaggrin, and linoleic acid showed the best fit orientation to bind filaggrin. So, generally, both oleic and linoleic acids have the potential to bind filaggrin well as a drug in atopic dermatitis treatment. Besides having the most stable interaction with filaggrin, oleic and palmitic acids have same binding side with filaggrin. The same binding sides between oleic acid with filaggrin and palmitic acid (control ligand) with filaggrin 
showed the fit medical interaction between oleic acid and filaggrin. Both the interaction stability and the fit interaction between oleic acid and filaggrin raise the effectiveness of oleic acid as a drug in atopic dermatitis treatment. The bond between oleic acid and filaggrin was formed via hydrophobic interaction on LEU D75. Hydrophobic interactions generated by oleic acid caused the displacement of water molecules so that the entropy value is changed. The interaction between oleic acid and amino acid residues resulted in an increase in the entropy value which drives (catalyzing) the interaction between the protein and ligand. Therefore, hydrophobic interactions have an important role in the ligand and protein binding [21].

In comparison, a placebo-controlled study [22] has been conducted to investigate the effects of seed oil and pulp oil from sea buckthorn (Hippophae rhamnoides) in atopic dermatitis patients. The concentration of oleic acid detected is $19 \%$ as the main fatty acid in seed oil and $26 \%$ in pulp oil. The results showed that there were no changes in triacylglycerol levels, serum total, or specific immunoglobulin E detected. However, Fellermeier [23] who assessed the relationship between intake of selected foods and fatty acids with the prevalence of atopic disease in adults showed that in women, high total fat, palmitoleic acid, and oleic acid intake was positively associated with sensitization. High total fat consumption, high monounsaturated fatty acids, and high oleic acid are positively associated with fever. Thus, the oleate present in this oil is more appropriate for oral use. Ferreri et al. [24] reinforce that radical stress is related to the inflammatory process of atopic disease which is directly related to trans-FA (fatty acids) detected in patients with this treatment. Trans lipid isomers were found in both layers of the skin cell membrane, with a sequential total content of $2.7 \%$ and $4.9 \%$ of the FA composition. Using an in vitro model, trans lipid isomers were detected by the isomerization of thiyl radicals-catalysts, namely, the appearance of oleic and arachidonic acid isomers. This study shows that oleic acid is appropriate for atopic dermatitis in terms of nonoral aspects of in vivo reaction because it does not cause an immune response (e.g., the appearance of immunoglobulin E).

The epidermis plays an important role in protecting human subjects from exogenous stressors and helps maintain internal fluid and electrolyte homeostasis [25]. Thus, the appropriate epidermal structure will affect the physical, chemical, biochemical, and immune functions of the skin effectively. Filaggrin, one of the epidermal proteins, is very important for the formation of corneocytes and intracellular metabolites, which in turn contribute to maintaining stratum corneum moisture and acidic surface of the skin [26].

Filaggrin is an epidermal protein that is important in controlling the hydration and stratum corneum $\mathrm{pH}$ [27]. In patients with a deficiency of filaggrin, it is characterized by the appearance of atopic dermatitis, suggesting that the absence of filaggrin is a key factor in the pathogenesis of this skin condition [28]. Atopic dermatitis is a skin disorder in the form of chronic inflammation characterized by a defect in the epidermal barrier and keratinocyte differentiation.
Filaggrin expression is vital because this protein is considered to have a major role in epidermal function [26]. Thus, oleic is a material that is compatible with filaggrin so that it is suitable and has the potential to cure patients/patients with atopic dermatitis who experience impaired skin epidermal function.

\section{Conclusions}

Pliek $U$ oil has been successfully analyzed by GC-MS which showed that lauric acid, myristic acid, palmitic acid, and oleic acid were the predominant fatty acid contents. Based on the molecular docking analysis, oleic acid showed the most stable interaction with filaggrin with the lowest binding affinity $(-6.1 \mathrm{kcal} / \mathrm{mol})$ compared with others. Oleic and palmitic acids also have one same side binding to filaggrin on amino acid LEU D75. Therefore, oleic acid had the best potential to be used as a drug candidate in atopic dermatitis treatment.

\section{Data Availability}

Data used in this research have not been made available because they are supplementary data and will be used in future work.

\section{Conflicts of Interest}

The authors declare no conflicts of interest.

\section{Acknowledgments}

The authors wish to thank the Department of Biology, Faculty of Mathematics and Natural Sciences, Universitas Negeri Malang, and the Department of Chemistry, Faculty of Mathematics and Natural Sciences, Universitas Syiah Kuala, for allocation of facilities during this study.

\section{References}

[1] A. Sandilands, C. Sutherland, A. D. Irvine, and W. H. McLean, "Filaggrin in the frontline: role in skin barrier function and disease," Journal of Cell Science, vol. 122, no. 9, pp. 1285-1294, 2009.

[2] J. van Smeden, M. Janssens, E. C. J. Kaye et al., "The importance of free fatty acid chain length for the skin barrier function in atopic eczema patients," Experimental Dermatology, vol. 23, no. 1, pp. 45-52, 2014.

[3] Y. Ding, X. Shao, X. Li et al., "Identification of candidate genes in atopic dermatitis based on bioinformatic methods," International Journal of Dermatology, vol. 55, no. 7, pp. 791-800, 2016.

[4] C. N. A. Palmer, A. D. Irvine, A. Terron-Kwiatkowski et al., "Common loss-of-function variants of the epidermal barrier protein filaggrin are a major predisposing factor for atopic dermatitis," Nature Genetics, vol. 38, no. 4, pp. 441-446, 2006.

[5] K. M. Mogensen, "Essential fatty acid deficiency," Practical Gastroenterology, vol. 37, 2017.

[6] L. Guenther, C. W. Lynde, A. Andriessen et al., "Pathway to dry skin prevention and treatment," Journal of Cutaneous Medicine and Surgery, vol. 16, no. 1, pp. 23-31, 2012. 
[7] A. R. Vaughn, A. K. Clark, R. K. Sivamani, and V. Y. Shi, "Natural oils for skin-barrier repair: ancient compounds now backed by modern science," American Journal of Clinical Dermatology, vol. 19, no. 1, pp. 103-117, 2018.

[8] T.-K. Lin, L. Zhong, and J. Santiago, "Anti-inflammatory and skin barrier repair effects of topical application of some plant oils," International Journal of Molecular Sciences, vol. 19, no. 1, p. 70, 2017.

[9] N. M. Noor, "The effect of virgin coconut oil loaded solid lipid particles (VCO-SLPs) on skin hydration and skin elasticity," Jurnal Teknologi, vol. 62, no. 1, 2013.

[10] B White, "Dietary fatty acids," American Family Physician, vol. 80, no. 4, pp. 345-350, 2009.

[11] L. R. Laureles, F. M. Rodriguez, C. E. Reaño, G. A. Santos, A. C. Laurena, and E. M. T. Mendoza, "Variability in fatty acid and triacylglycerol composition of the oil of coconut (cocos nucifera L.) hybrids and their parentals," Journal of Agricultural and Food Chemistry, vol. 50, no. 6, pp. 1581-1586, 2002.

[12] E. V. Carandang, "Health benefits of virgin coconut oil," vol. 38, no. 9, 2008.

[13] E. Suhartono, I. Thalib, I. Aflanie, Z. Noor, and R. Idroes, "Study of interaction between cadmium and bovine serum albumin with UV-vis spectrocopy approach," IOP Conference Series: Materials Science and Engineering, vol. 350, Article ID 012008, 2018.

[14] U. Hasanah, M. Setyowati, R. Efendi et al., "Preparation and characterization of a pectin membrane-based optical $\mathrm{pH}$ sensor for fish freshness monitoring," Biosensors, vol. 9, no. 2, p. 60, 2019.

[15] K. Lahna, R. Idroes, N. Idris et al., "Formation and emission characteristics of $\mathrm{CN}$ molecules in laser induced low pressure He plasma and its applications to $\mathrm{N}$ analysis in coal and fossilization study," Applied Optics, vol. 55, no. 7, p. 1731, 2016.

[16] R. Hedwig, K. Lahna, R. Idroes et al., "Food analysis employing high energy nanosecond laser and low pressure $\mathrm{He}$ ambient gas," Microchemical Journal, vol. 147, pp. 356-364, 2019.

[17] M. R. Hurle, L. Yang, Q. Xie, D. K. Rajpal, P. Sanseau, and P. Agarwal, "Computational drug repositioning: from data to therapeutics," Clinical Pharmacology \& Therapeutics, vol. 93, no. 4, pp. 335-341, 2013.

[18] N. Earlia, R. Rahmad, M. Amin, C. Prakoeswa, K. Khairan, and R. Idroes, "The potential effect of fatty acids from pliek $U$ on epidermal fatty acid binding protein: chromatography and bioinformatic studies," Sains Malaysiana, vol. 48, no. 5, pp. 1019-1024, 2019.

[19] T. Cheng, Q. Li, Z. Zhou, Y. Wang, and S. H. Bryant, "Structure-based virtual screening for drug discovery: a problem-centric review," The AAPS Journal, vol. 14, no. 1, pp. 133-141, 2012.

[20] J. Orsavova, L. Misurcova, J. Ambrozova, R. Vicha, and J. Mlcek, "Fatty acids composition of vegetable oils and its contribution to dietary energy intake and dependence of cardiovascular mortality on dietary intake of fatty acids," International Journal of Molecular Sciences, vol. 16, no. 6, pp. 12871-12890, 2015.

[21] C. E. Tinberg, S. D. Khare, J. Dou et al., "Computational design of ligand-binding proteins with high affinity and selectivity," Nature, vol. 501, no. 7466, pp. 212-216, 2013.

[22] B. Yang, "Effects of dietary supplementation with sea buckthorn (Hippophaë rhamnoides) seed and pulp oils on atopic dermatitis," The Journal of Nutritional Biochemistry, vol. 10, no. 11, pp. 622-630, 1999.
[23] M. A. Trak-Fellermeier, S. Brasche, G. Winkler, B. Koletzko, and J. Heinrich, "Food and fatty acid intake and atopic disease in adults," European Respiratory Journal, vol. 23, no. 4, pp. 575-582, 2004.

[24] C. Ferreri, F. Angelini, C. Chatgilialoglu et al., "Trans fatty acids and atopic eczema/dermatitis syndrome: the relationship with a free radical cis-trans isomerization of membrane lipids," Lipids, vol. 40, no. 7, pp. 661-667, 2005.

[25] J. P. Thyssen and S. Kezic, "Causes of epidermal filaggrin reduction and their role in the pathogenesis of atopic dermatitis," Journal of Allergy and Clinical Immunology, vol. 134, no. 4, pp. 792-799, 2014.

[26] V. Pendaries, M. Le Lamer, L. Cau et al., "In a three-dimensional reconstructed human epidermis filaggrin-2 is essential for proper cornification," Cell Death \& Disease, vol. 6, no. 2, p. e1656, 2015.

[27] V. Pendaries, J. Malaisse, L. Pellerin et al., "Knockdown of filaggrin in a three-dimensional reconstructed human epidermis impairs keratinocyte differentiation," Journal of Investigative Dermatology, vol. 134, no. 12, pp. 2938-2946, 2014.

[28] T. Fukuie, R. Yasuoka, T. Fujiyama, J. Sakabe, T. Taguchi, and Y. Tokura, "Palmar hyperlinearity in early childhood atopic dermatitis is associated with filaggrin mutation and sensitization to egg," Pediatric Dermatology, vol. 36, no. 2, pp. 213-218, 2019. 


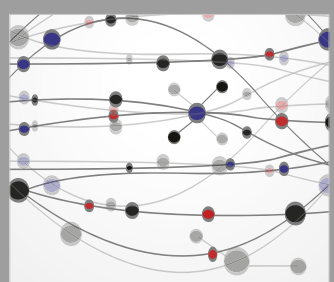

The Scientific World Journal
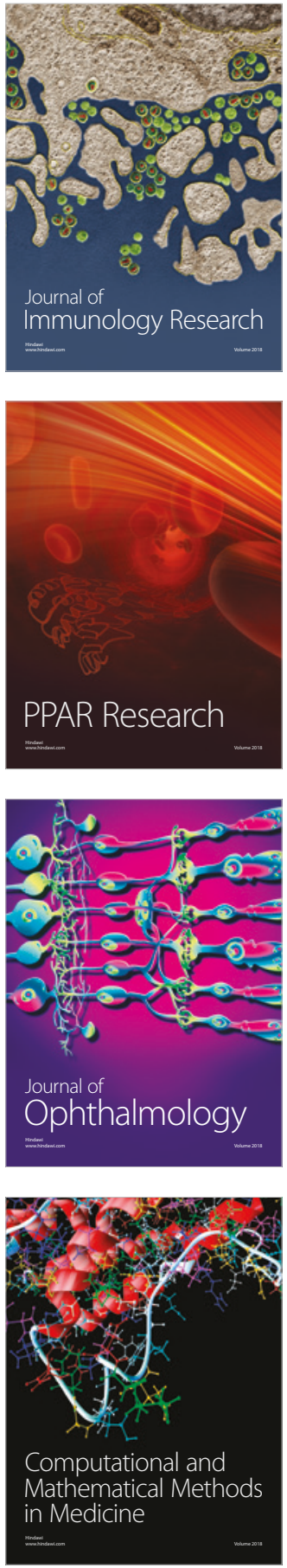

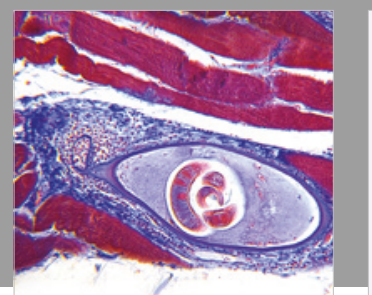

Gastroenterology Research and Practice

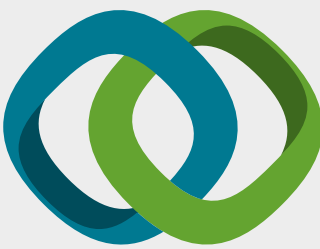

\section{Hindawi}

Submit your manuscripts at

www.hindawi.com
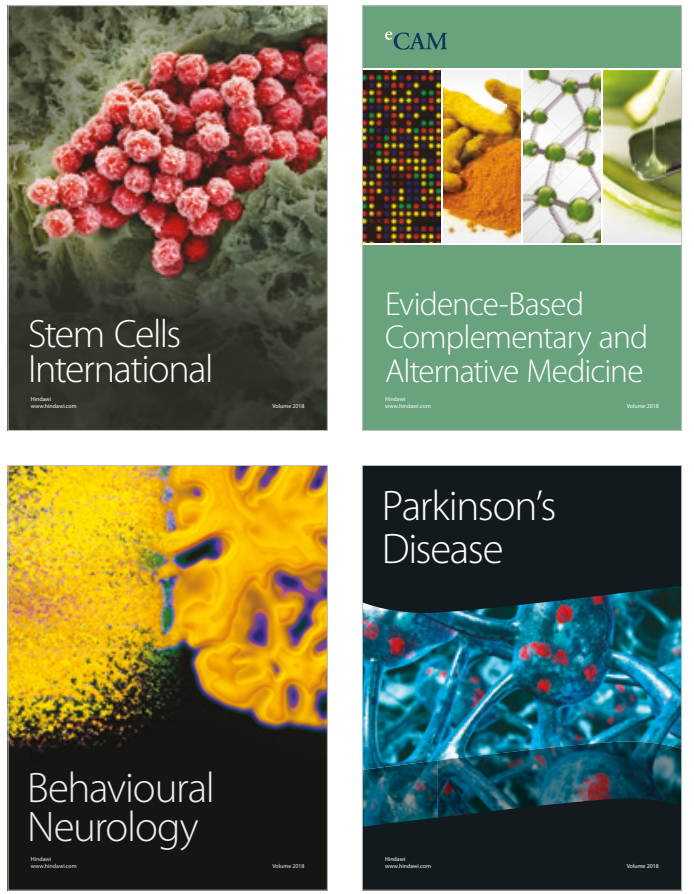

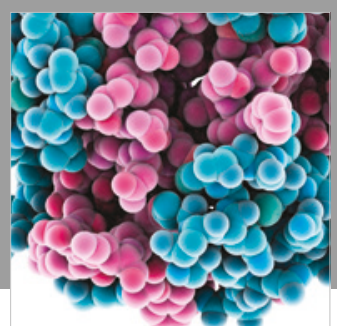

ournal of

Diabetes Research

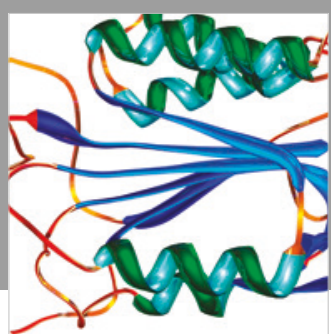

Disease Markers
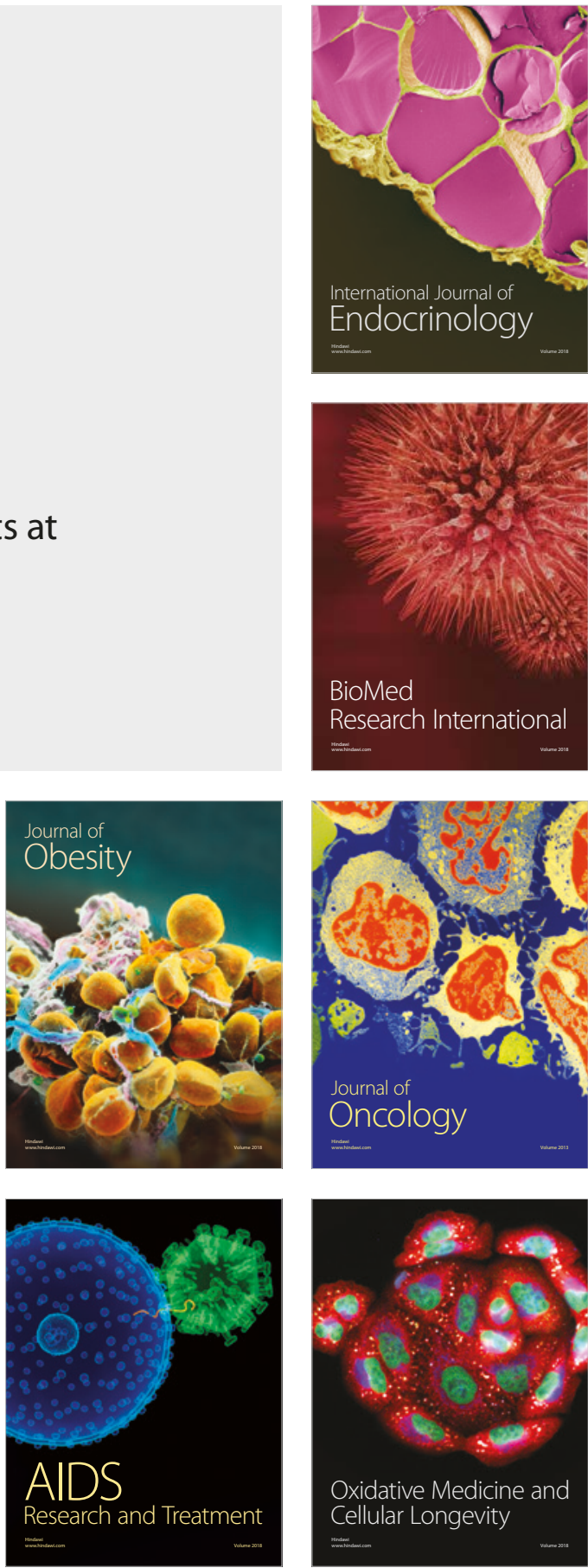